\title{
Muscle Weakness Upper Limb, CTCAE
}

National Cancer Institute

\section{Source}

National Cancer Institute. Muscle Weakness Upper Limb, CT CAE. NCI Thesaurus. Code C143685.

A disorder characterized by a reduction in the strength of the upper limb muscles. 